\title{
Distributed Frequency Control with Operational Constraints, Part II: Network Power Balance
}

\author{
Zhaojian Wang, Feng Liu, Steven H. Low, Fellow, IEEE, Changhong Zhao, and Shengwei Mei Fellow, IEEE
}

\begin{abstract}
In Part I of this paper we propose a decentralized optimal frequency control of multi-area power system with operational constraints, where the tie-line powers remain unchanged in the steady state and the power mismatch is balanced within individual control areas. In Part II of the paper, we propose a distributed controller for optimal frequency control in the network power balance case, where the power mismatch is balanced over the whole system. With the proposed controller, the tieline powers remain within the acceptable range at equilibrium, while the regulation capacity constraints are satisfied both at equilibrium and during transient. It is revealed that the closedloop system with the proposed controller carries out primal-dual updates with saturation for solving an associated optimization problem. To cope with discontinuous dynamics of the closed-loop system, we deploy the invariance principle for nonpathological Lyapunov function to prove its asymptotic stability. Simulation results are provided to show the effectiveness of our controller.
\end{abstract}

Index Terms-Power system dynamics, frequency control; network power balance; distributed control.

\section{INTRODUCTION}

In Part I of the paper we have investigated the optimal frequency control of multi-area power system with operational constraints [1]. In that case, the tie-line powers are required to be unchanged in the steady state after load disturbances, which implies the power mismatch in each area has to be balanced individually. It is referred to as the per-node power balance case. In Part II of the paper, we consider the transmission congestion in the distributed optimal frequency design.

The per-node balance case in Part I mainly considers the situation where the power delivered from one area to another is fixed, e.g. contract power, which should not be violated in normal operation. However, in some circumstances, control areas may cooperate for better frequency recovery or regulation cost reduction. In this case, power mismatch may be balanced by all generations and controllable loads among all control areas in cooperation. Similar situations also appear in one control area with multiple generators and controllable loads that cooperate to eliminate the power mismatch in the area. It is referred to as the network power balance case. Compared with the

This work was supported by the National Natural Science Foundation of China ( No. 51377092, No. 51677100, No. 51621065), Foundation of Chinese Scholarship Council (CSC No. 201506215034), the US National Science Foundation through awards EPCN 1619352, CCF 1637598, CNS 1545096 , ARPA-E award DE-AR0000699, and Skoltech through Collaboration Agreement 1075-MRA.

Z. Wang, F. Liu and S. Mei are with the Department of Electrical Engineering, Tsinghua University, Beijing, China, 100084 e-mail: (lfeng@tsinghua.edu.cn).

S. H. Low and C. Zhao are with the Department of Electrical Engineering, California Institute of Technology, Pasadena, CA, USA, 91105 email:(slow@caltech.edu) per-node balance case, the most challenging problem in this case is that the tie-line powers may change and congestions may occur. In addition, local information is not sufficient and neighboring information turns to be helpful. As for the constraints, the tie-line power constraints are not hard limits, which only need to be satisfied at equilibrium. The capacity limits on the generations and controllable loads also required to be satisfied both in steady state and during transient.

In the recent literature of frequency control, tie-line power constraints are considered in [2]-[7]. In [2], tie-line power constraints are included in the load-side secondary frequency control. A virtual variable is used to estimate the tie-line power, whose value is identical to the tie-line power at equilibrium. In [3], [4], an optimal economic dispatch problem including tie-line power constraints is formulated, then the solution dynamics derived from a primal-dual algorithm is shaped as a port-Hamiltonian form. The power system dynamics also have a port-Hamiltonian form, which are interconnected with the solution dynamics to constitute a closedloop Hamiltonian system. Then, the optimality and stability are proved. In [6], a unified method is proposed for primary and secondary frequency control, where the congestion management is implemented in the secondary control. In [7], a realtime control framework is proposed for tree power networks, where transmission capacities are considered.

Similar to the per-node balance case, hard limits, such as capacity constraints of power injections on buses, are enforced only in the steady-state in the literature, which may fail if such constraints are violated in transient. Here we construct a fully distributed control to recover nominal frequency while eliminating congestion. Differing from the literature, it enforces regulation capacity constraints not only at equilibrium, but also during transient. We show that the controllers together with the physical dynamics serve as primal-dual updates with saturation for solving the optimization problem. The optimal solution of the optimization problem and the equilibrium point of closed-loop system are identical.

The enforcement of capacity constraints during transient and tie-line power limits simultaneously makes the stability proof difficult. Specifically, the Lyapunov function is not continuous anymore, as in the per-node case in Part I of the paper. In this situation, the conventional LaSalle's invariance principle does not apply. To overcome the difficulty, we construct a nonpathological Lyapunov function to mitigate the impacts of nonsmooth dynamics. The salient features of the controller are:

1) Control goals: the controller restores the nominal frequency and balance the power mismatch in the whole 
system after unknown load disturbance while minimizing the regulation costs;

2) Constraints: the regulation capacity constraints are always enforced even during transient and the congestions can be eliminated automatically;

3) Communication: only neighborhood communication is needed in the network balance case;

4) Measurement: the controller is adaptive to unknown load disturbances automatically with no need of load measurement.

The rest of this paper is organized as follows. In Section II, we describe our model. Section III formulates the optimal frequency control problem in the network balance case, presents the distributed frequency controller and proves the optimality, uniqueness and stability of the closed-loop equilibrium. Simulation results are given in Section IV. Section $\mathrm{V}$ concludes the paper.

\section{NETWORK MODEL}

We summarize the notation used in Part I [1]. The power network is model by a directed graph $G:=(N, E)$ where $N=$ $\{0,1,2, \ldots n\}$ is the set of nodes (control areas) and $E \subseteq N \times$ $N$ is the set of edges (tie lines). If a pair of nodes $i$ and $j$ are connected by a tie line directly, we denote the tie line by $(i, j) \in E$. Let $m:=|E|$ denote the number of tie lines. Use $(i, j) \in E$ or $i \rightarrow j$ interchangeably to denote a directed edge from $i$ to $j$. Assume the graph is connected and node 0 is a reference node. For each node $j \in N, \theta_{j}(t)$ denotes the rotor angle at node $j$ at time $t$ and $\omega_{j}(t)$ is the frequency. $P_{j}^{g}(t)$ denotes the (aggregate) generation at node $j$ at time $t$ and $u_{j}^{g}(t)$ is its generation control command. $P_{j}^{l}(t)$ denotes the (aggregate) controllable load and $u_{j}^{l}(t)$ is its load control command. $p_{j}$ is the (aggregate) uncontrollable load.

The power system dynamics for each node $j \in N$ is

$$
\begin{aligned}
\dot{\theta}_{j} & =\omega_{j}(t) \\
M_{j} \dot{\omega}_{j} & =P_{j}^{g}(t)-P_{j}^{l}(t)-p_{j}-D_{j} \omega_{j}(t) \\
& +\sum_{i: i \rightarrow j} B_{i j}\left(\theta_{i}(t)-\theta_{j}(t)\right)-\sum_{k: j \rightarrow k} B_{j k}\left(\theta_{j}(t)-\theta_{k}(t)\right) \\
T_{j}^{g} \dot{P}_{j}^{g} & =-P_{j}^{g}(t)+u_{j}^{g}(t)-\omega_{j}(t) / R_{j} \\
T_{j}^{l} \dot{P}_{j}^{l} & =-P_{j}^{l}(t)+u_{j}^{l}(t)
\end{aligned}
$$

where $D_{j}>0$ are damping constants, $R_{j}>0$ are droop parameters, and $B_{j k}>0$ are line parameters that depend on the reactance of the line $(j, k)$. Let $x:=\left(\theta, \omega, P^{g}, P^{l}\right)$ denote the state of the network and $u:=\left(u^{g}, u^{l}\right)$ denote the control.

The capacity constraints are:

$$
\begin{aligned}
& \underline{P}_{j}^{g} \leq P_{j}^{g}(t) \leq \bar{P}_{j}^{g}, \quad j \in N \\
& \underline{P}_{j}^{l} \leq P_{j}^{l}(t) \leq \bar{P}_{j}^{l}, \quad j \in N
\end{aligned}
$$

Here (2a) and (2b) are hard limits on the regulation capacities of generation and controllable load at each node, which should not be violated at any time even during transient.

The system operates in a steady state initially, i.e., the generation and the load are balanced and the frequency is at its nominal value. All variables represent deviations from their nominal or scheduled values so that, e.g., $\omega_{j}(t)=0$ means the frequency is at its nominal value.

In this paper, all nodes cooperate to rebalance power over the entire network after a disturbance. The power flows $P_{i j}$ on the tie lines may deviate from their scheduled values and we require that they satisfy line limits, i.e.,

$$
\underline{P}_{i j} \leq P_{i j} \leq \bar{P}_{i j} \quad \forall(i, j) \in E
$$

for some upper and lower bounds $\underline{P}_{i j}, \bar{P}_{i j}$.

In DC approximation the power flow on line $(i, j)$ is given by $P_{i j}=B_{i j}\left(\theta_{i}-\theta_{j}\right)$. Hence line flow constraints in the pernode balance case is $\theta_{i}=\theta_{j}$ for all $(i, j) \in E$ and in the network balance case is:

$$
\underline{\theta}_{i j} \leq \theta_{i}-\theta_{j} \leq \bar{\theta}_{i j} \quad \forall(i, j) \in E
$$

where $\underline{\theta}_{i j}=\underline{P}_{i j} / B_{i j}, \bar{\theta}_{i j}=\bar{P}_{i j} / B_{i j}$ are lower and upper bounds on angle differences.

As the generation $P_{j}^{g}$ and load $P_{j}^{l}$ in each area can increase or decrease, and a line flow $P_{i j}$ can in either direction, we make the following assumption.
A1:
1) $\underline{P}_{j}^{g}<0<\bar{P}_{j}^{g}$ and $\underline{P}_{j}^{l}<0<\bar{P}_{j}^{l}$ for $\forall j \in N$.
2) $\underline{\theta}_{i j} \leq 0 \leq \bar{\theta}_{i j}$ for $(i, j) \in E$.
3) $\theta_{0}(t):=0$ and $\phi_{0}(t):=0$ for all $t \geq 0$.

Here $\phi$ is a vector variable that represents virtual phase angles in the network balance case in Section III. The assumption $\theta_{0} \equiv \phi_{0} \equiv 0$ amounts to using $\left(\theta_{0}(t), \phi_{0}(t)\right)$ as reference angles. It is made merely for notational convenience: as we will see, the equilibrium point will be unique with this assumption (or unique up to reference angles without this assumption).

\section{Controller Design for The Network Power BALANCE CASE}

In the per-node balance case, individual control areas rebalance power within their own areas after disturbances. However, in many circumstances, it may be more efficient for all control areas to eliminate power imbalance of the overall system in a coordinated manner. This can be modeled as the condition:

$$
\sum_{j} P_{j}^{g}=\sum_{j}\left(P_{j}^{l}+p_{j}\right)
$$

In this case the tie-line flows may not be restored to their predisturbance values. To ensure that they are within operational limits, the constraints (3) are imposed.

Even though the philosophy of the controller design as well as the proofs are similar to the per-node case, the details are much more complicated. Our presentation will however be brief where there is no confusion. 


\section{A. Control goals}

In the network power balance case, the control goals are formalized as the following optimization problem.

NBO: $\min \quad \sum_{j} \frac{\alpha_{j}}{2}\left(P_{j}^{g}\right)^{2}+\sum_{j} \frac{\beta_{j}}{2}\left(P_{j}^{l}\right)^{2}+\sum_{j} \frac{D_{j}}{2} \omega_{j}^{2}+\sum_{j} \frac{z_{j}^{2}}{2}$

over $\quad x:=\left(\theta, \phi, \omega, P^{g}, P^{l}\right)$ and $u:=\left(u^{g}, u^{l}\right)$

s. t. (2),

$$
\begin{aligned}
& P_{j}^{g}=P_{j}^{l}+p_{j}+U_{j}(\theta, \omega) \quad j \in N \\
& P_{j}^{g}=P_{j}^{l}+p_{j}+\hat{U}_{j}(\phi) \quad j \in N \\
& \underline{\theta}_{i j} \leq \phi_{i}-\phi_{j} \leq \bar{\theta}_{i j}, \quad(i, j) \in E \\
& P_{j}^{g}=u_{j}^{g}, \quad j \in N \\
& P_{j}^{l}=u_{j}^{l}, \quad j \in N
\end{aligned}
$$

where $\alpha_{j}>0, \beta_{j}>0$ are constant weights; $z_{j}$ is a shorthand defined for convenience as

$$
z_{j}:=P_{j}^{g}-P_{j}^{l}-p_{j}-\hat{U}_{j}(\phi)
$$

$U(\theta, \omega):=D \omega+C B C^{T} \theta$, and $\hat{U}(\phi):=C B C^{T} \phi$.

While $\theta$ represents the phase angles in the physical power network, $\phi$ is a cyber quantity that can be interpreted as virtual phase angles (see remarks below). The matrices $D, C$ and $B$ are defined as in the previous section.

As in the per-node case, we define the variables $\tilde{\theta}_{i j}:=\theta_{i}-$ $\theta_{j}$, or in vector form, $\tilde{\theta}:=C^{T} \theta$. As we fix $\theta_{0}:=0$ to be a reference angle under assumption $\mathrm{A} 1, \tilde{\theta}=C^{T} \theta$ defines a bijection between $\theta$ and $\tilde{\theta}$. Similarly we define $\tilde{\phi}_{i j}:=\phi_{i}-\phi_{j}$ or $\tilde{\phi}:=C^{T} \phi$, and $\phi_{0}:=0$ so there is a bijection between $\phi$ and $\tilde{\phi}$. Note that both $\tilde{\theta}$ and $\tilde{\phi}$ are restricted to the column space of $C^{T}$. We will use $(\theta, \phi)$ and $(\tilde{\theta}, \tilde{\phi})$ interchangeably. For instance we will abuse notation and write $\hat{U}(\phi):=C B C^{T} \phi$ or $\hat{U}(\tilde{\phi}):=C B \tilde{\phi}$.

We now summarize some of the interesting properties of NBO (5) that will be proved formally in the next two subsections. We first compare NBO (5) with PBO in [1] for the per-node balance case.

Remark 1 (Comparison of NBO and PBO). 1) Intuitively the network balance condition (4) is a relaxation of the per-node balance condition (3) in [1], and hence we expect that the optimal cost of NBO lower bounds that of PBO. This is indeed the case, as we now argue. Constraint (5c) implies that any feasible point of (5) has $z_{j}=0$ and hence these two optimization problems have the same objective function. Their variables and constraints are different in that PBO directly enforces the per-node balance condition while NBO (5) has the additional variable $\phi$ and constraints (5c)(5d). Any optimal point $\left(\theta^{*}, \omega^{*}, P^{g^{*}}, P^{l^{*}}\right)$ for PBO however defines a feasible point $\left(\theta^{*}, \phi, \omega^{*}, P^{g *}, P^{l *}\right)$ for NBO (5) with the same cost where $\phi=\theta^{*}$. The point $\left(\theta^{*}, \phi, \omega^{*}, P^{g *}, P^{l *}\right)$ satisfies $(5 \mathrm{c})(5 \mathrm{~d})$ because $\left(\theta^{*}, \omega^{*}, P^{g *}, P^{l^{*}}\right)$ satisfies $(5 \mathrm{~b}), \omega^{*}=0$ and $\theta_{i}^{*}=\theta_{j}^{*}$ by Theorem 2 in [1], and $\underline{\theta}_{i j} \leq 0 \leq \bar{\theta}_{i j}$ by assumption A1.
2) Even though any feasible point of (5) has $z_{j}=0$, the objective function is augmented with $z_{j}^{2}$ to improve convergence (see [8]).

Even though neither the network balance condition (4) nor the line limits (3) are explicitly enforced in (5), they are satisfied at optimality (Theorem 2 below). Indeed, the virtual phase angles $\phi$ and the conditions (5b)-(5d) are carefully designed to enforce these conditions as well as to restore the nominal frequency $\omega^{*}=0$ at optimality. This technique is previously used in [2].

Remark 2 (Virtual phase angles $\phi$ ). 1) Under mild conditions, $\omega^{*}=0$ at optimality for both PBO and NBO. For NBO, this is a consequence of the constraint $(5 \mathrm{c})$ on $\phi$; see Lemma A.1. Summing (5c) over all $j \in N$ also implies the network balance condition (4) since $\mathbf{1}^{T} \hat{U}(\phi)=\mathbf{1}^{T} C B C^{T} \phi=0$.

2) In PBO, $\theta_{i}^{*}=\theta_{j}^{*}$ at optimality (i.e., tie-line flows are restored $P_{i j}^{*}=0$ ) and $U\left(\theta^{*}, \omega^{*}\right)=0$. This does not necessarily hold in NBO. However $\phi$ is regarded as virtual phase angles because, at optimality, $\phi^{*}$ differs from the real phase angles $\theta^{*}$ only by a constant, $\phi^{*}-\theta^{*}=\mathbf{1}\left(\phi_{0}-\theta_{0}\right)$ (Lemma A.1 in the appendix). Hence $C^{T} \phi^{*}-C^{T} \theta^{*}=C^{T} \cdot \mathbf{1}\left(\phi_{0}-\theta_{0}\right)=0$, implying $\tilde{\phi}_{i j}^{*}=\tilde{\theta}_{i j}^{*}$. Then the constraints (5d) are exactly the flow constraints (3). In other words, we impose the flow constraints on $\tilde{\theta}$ indirectly by enforcing such constraints on the virtual angle $\tilde{\phi}$. .

\section{B. Distributed controller}

Our control laws are:

$$
\begin{array}{crr}
\dot{\lambda}_{j}= & \gamma_{j}^{\lambda}\left(P_{j}^{g}(t)-P_{j}^{l}(t)-p_{j}-\hat{U}_{j}(\tilde{\phi}(t))\right), j \in N \\
\dot{\eta}_{i j}^{+}= & \gamma_{i j}^{\eta}\left[\tilde{\phi}_{i j}(t)-\bar{\theta}_{i j}\right]_{\eta_{i j}^{+}}^{+} & \forall(i, j) \in E \\
\dot{\eta}_{i j}^{-}= & \gamma_{i j}^{\eta}\left[\underline{\theta}_{i j}-\tilde{\phi}_{i j}(t)\right]_{\eta_{i j}^{-}}^{+} & \forall(i, j) \in E \\
\dot{\tilde{\phi}}_{i j}= & \gamma_{i j}{ }_{i j}\left(B_{i j}\left[\lambda_{i}(t)-\lambda_{j}(t)+z_{i}(t)-z_{j}(t)\right]\right. \\
& \left.+\eta_{i j}^{-}(t)-\eta_{i j}^{+}(t)\right) & \forall(i, j) \in E
\end{array}
$$

$$
\begin{aligned}
& u_{j}^{g}(t)= {\left[P_{j}^{g}(t)-\gamma_{j}^{g}\left(\alpha_{j} P_{j}^{g}(t)+\omega_{j}(t)+z_{j}(t)+\lambda_{j}(t)\right)\right]_{\underline{P}_{j}^{g}}^{\bar{P}_{j}^{g}} } \\
&+\omega_{j}(t) / R_{j}, \quad j \in N \quad(6 \mathrm{e}) \\
& u_{j}^{l}(t)= {\left[P_{j}^{l}(t)-\gamma_{j}^{l}\left(\beta_{j} P_{j}^{l}(t)-\omega_{j}(t)-z_{j}(t)-\lambda_{j}(t)\right)\right]_{\underline{P}_{j}^{l}}^{\bar{P}_{j}^{l}} } \\
& j \in N
\end{aligned}
$$

where $\gamma_{j}^{\lambda}, \gamma_{i j}^{\eta}, \gamma_{i j}^{\tilde{\phi}}, \gamma_{j}^{g}, \gamma_{j}^{l}$ are positive constants. For any $x_{i}, a_{i} \in$ $\mathbb{R}$, the operator $\left[x_{i}\right]_{a_{i}}^{+}$is defined by

$$
\left[x_{i}\right]_{a_{i}}^{+}:= \begin{cases}x_{i} & \text { if } a_{i}>0 \text { or } x_{i}>0 \\ 0, & \text { otherwise }\end{cases}
$$

For a vector case, $[x]_{a}^{+}$is defined accordingly componentwise [9]. 
Here we assume that each node $i$ updates a set of internal states $\left(\lambda_{i}(t), \eta_{i j}^{+}(t), \eta_{j i}^{-}(t), \tilde{\phi}_{i j}(t)\right)$ according to $(6 \mathrm{a})-(6 \mathrm{~d}) .{ }^{1}$ In contrast to the completely decentralized control derived in the per-node balance case, here the control is distributed where each node $i$ updates $\left(\lambda_{i}(t), \eta_{i j}^{+}(t), \eta_{i j}^{-}(t)\right)$ using only local measurements or computation but requires the information $\left(\lambda_{j}(t), z_{j}(t)\right)$ from its neighbors $j$ to update $\tilde{\phi}_{i j}(t)$. Note that $z_{i}(t)$ is not a variable but a shorthand for (function) $P_{j}^{g}(t)-$ $P_{j}^{l}(t)-p_{j}-\hat{U}_{j}(\tilde{\phi}(t))$. The control inputs $\left(u_{i}^{g}(t), u_{i}^{l}(t)\right)$ in (6e)(6f) are functions of the network state $\left(P_{i}^{g}(t), P_{i}^{l}(t), \omega_{i}(t)\right)$ and the internal state $\left(\lambda_{i}(t), \eta_{i j}^{+}(t), \eta_{i j}^{-}(t), \tilde{\phi}_{i j}(t)\right)$. We write $u_{j}^{g}$ and $u_{j}^{l}$ as functions of $\left(P_{j}^{g}, P_{j}^{l}, \omega_{j}, \lambda_{j}\right)$ : for $j \in N$

$$
\begin{aligned}
& u_{j}^{g}(t):=u_{j}^{g}\left(P_{j}^{g}(t), \omega_{j}(t), \lambda_{j}(t), z_{j}(t)\right) \\
& u_{j}^{l}(t):=u_{j}^{l}\left(P_{j}^{l}(t), \omega_{j}(t), \lambda_{j}(t), z_{j}(t)\right)
\end{aligned}
$$

where the functions are defined by the right-hand sides of (6e)(6f).

Now we comment on the implementation of the control (6).

Remark 3 (Implementation). 1) As discussed above, communication is needed only between neighboring nodes (areas) to update the variables $\tilde{\phi}_{i j}(t)$.

2) Similar to the per-node power balance case, we can avoid measuring the load change $p_{j}$ by using (1b) and the definition of $z_{j}(t)$ to replace (6a) with

$$
\begin{aligned}
z_{j}(t)= & M_{j} \dot{\omega}_{j}+D_{j} \omega_{j}(t)-\sum_{i: i \rightarrow j} P_{i j}(t)+\sum_{k: j \rightarrow k} P_{j k}(t) \\
& -\hat{U}_{j}(\tilde{\phi}(t)) \\
\dot{\lambda}_{j}= & \gamma_{j}^{\lambda} z_{j}(t)
\end{aligned}
$$

\section{Design rationale}

The controller design (6) is also motivated by a (partial) primal-dual algorithm for (5), as for the per-node power balance case.

Primal-dual algorithms. The optimization problem in the network balance case differs from that in the per-node balance case in the inequalities (5d) on $\tilde{\phi}$. Consider a general constrained convex optimization with inequality constraints:

$$
\min _{x \in X} f(x) \quad \text { s.t. } \quad g(x)=0, \quad h(x) \leq 0
$$

where $f: \mathbb{R}^{n} \rightarrow \mathbb{R}, g: \mathbb{R}^{n} \rightarrow \mathbb{R}^{k_{1}}, h: \mathbb{R}^{n} \rightarrow \mathbb{R}^{k_{2}}$, and $X \subseteq \mathbb{R}^{n}$ is closed and convex. Here an inequality constraint $h(x) \leq 0$ is imposed explicitly. Let $\rho_{1} \in \mathbb{R}^{k_{1}}$ be the Lagrange multiplier associated with the equality constraint $g(x)=0, \rho_{2} \in \mathbb{R}^{k_{2}}$ that associated with the inequality constraint $h(x) \leq 0$, and $\rho:=$ $\left(\rho_{1}, \rho_{2}\right)$. Define the Lagrangian $L(x ; \rho):=f(x)+\rho_{1}^{T} g(x)+$ $\rho_{2}^{T} h(x)$. A standard primal-dual algorithm takes the form:

$$
\begin{aligned}
x(t+1) & :=\operatorname{Proj}_{X}\left(x(t)-\Gamma^{x} \nabla_{x} L(x(t) ; \rho(t))\right) \\
\rho_{1}(t+1) & :=\rho_{1}(t)+\Gamma^{\rho_{1}} \nabla_{\rho_{1}} L(x(t) ; \rho(t)) \\
\rho_{2}(t+1) & :=\left(\rho_{2}(t)+\Gamma^{\rho_{2}} \nabla_{\rho_{2}} L(x(t) ; \rho(t))\right)^{+}
\end{aligned}
$$

${ }^{1}$ For each (directed) link $(i, j) \in E$ we assume that only node $i$ maintains the variables $\left(\eta_{i j}^{+}(t), \eta_{j i}^{-}(t), \tilde{\phi}_{i j}(t)\right)$. In practice, node $j$ will probably maintain symmetric variables to reduce communication burden or for other reasons outside our mathematical model here. where $\Gamma^{x}, \Gamma^{\rho_{1}}, \Gamma^{\rho_{2}}$ are strictly positive diagonal gain matrices. Here, if $a$ is a scalar then $(a)^{+}:=\max \{a, 0\}$ and if $a$ is a vector then $(a)^{+}$is defined accordingly componentwise. For a dual algorithm, (8a) is replaced by

$$
x(t):=\min _{x \in X} L(x ; \rho(t))
$$

As for the per-node balance case, all variables in $x(t)$ are updated according to (8a) except $\omega(t)$ which is updated according to ( $8 \mathrm{~d})$, as we see below.

The set $X$ in (8a) is defined by the constraints (2):

$$
X:=\left\{\left(P^{g}, P^{l}\right):\left(\underline{P}^{g}, \underline{P}^{l}\right) \leq\left(P^{g}, P^{l}\right) \leq\left(\bar{P}^{g}, \bar{P}^{l}\right)\right\}
$$

Controller (6) design. Let $\rho_{1}:=(\lambda, \mu)$ be the Lagrange multipliers associated with constraints $(5 \mathrm{c})$ and $(5 \mathrm{~b})$ respectively, $\rho_{2}:=\left(\eta^{+}, \eta^{-}\right)$the multipliers associated with constraints (5d), and $\rho:=\left(\rho_{1}, \rho_{2}\right)$. Define the Lagrangian of (5) by (10). Note that it is only a function of $(x, \rho)$ and independent of $u:=\left(u^{g}, u^{l}\right)$ as we treat $u$ as a function of $(x, \rho)$ defined by the right-hand sides of $(6 \mathrm{e})(6 \mathrm{f})$.

The closed-loop dynamics (1)(6) carry out an approximate primal-dual algorithm (8) for solving (5) in real time over the coupled physical power network and cyber computation. Since the reasoning is similar to the per-node balance case, we only provide a summary. Rewrite the Lagrangian $L_{2}$ in vector form

$$
\begin{aligned}
L_{2}(x ; \rho)= & \frac{1}{2}\left(\left(P^{g}\right)^{T} A^{g} P^{g}+\left(P^{l}\right)^{T} A^{l} P^{l}+\omega^{T} D \omega+z^{T} z\right) \\
& +\lambda^{T}\left(P^{g}-P^{l}-p-C B \tilde{\phi}\right) \\
& +\mu^{T}\left(P^{g}-P^{l}-p-D \omega-C B \tilde{\theta}\right) \\
& +\left(\eta^{+}\right)^{T}(\tilde{\phi}-\bar{\theta})+\left(\eta^{-}\right)^{T}(\underline{\theta}-\tilde{\phi})
\end{aligned}
$$

where $A^{l}:=\operatorname{diag}\left(\beta_{j}, j \in N\right), B:=\operatorname{diag}\left(B_{i j},(i, j) \in E\right)$.

First, the control $(6 \mathrm{~b})(6 \mathrm{c})$ can be interpreted as a continuoustime version of the dual update $(8 \mathrm{c})$ on the dual variable $\rho_{2}:=$ $\left(\eta^{+}(t), \eta^{-}(t)\right)$

$$
\begin{aligned}
& \dot{\eta}^{+}=\Gamma^{\eta}\left[\nabla_{\eta^{+}} L_{2}(x(t) ; \rho(t))\right]_{\eta^{+}(t)}^{+} \\
& \dot{\eta}^{-}=\Gamma^{\eta^{-}}\left[\nabla_{\eta^{-}} L_{2}(x(t) ; \rho(t))\right]_{\eta^{-}}^{+}
\end{aligned}
$$

where $\Gamma^{\eta}:=\operatorname{diag}\left(\gamma_{i j}^{\eta},(i, j) \in E\right)$.

Second, the control (6a) carries out the dual update (8b) on $\lambda(t)$ :

$$
\dot{\lambda}=\Gamma^{\lambda} \nabla_{\lambda} L_{2}(x(t), \rho(t))
$$

where $\Gamma^{\lambda}:=\operatorname{diag}\left(\gamma_{j}^{\lambda}, j \in N\right)$. The swing dynamic (1b) carries out the dual update (8b) on $\mu(t)$ because, as in the per-node balance case, we can identify $\mu(t) \equiv \omega(t)$ so that

$$
\dot{\mu}=\dot{\omega}=M^{-1} \nabla_{\mu} L_{2}(x(t) ; \rho(t))
$$

where $M:=\operatorname{diag}\left(M_{j}, j \in N\right)$.

Finally we show that (1a), (1c), (1d), and (6d) implement a mix of the primal updates (8a) and (8d) on the primal variables $x:=\left(\tilde{\theta}(t) ; \tilde{\phi}(t) ; \omega(t) ; P^{g}(t) ; P^{l}(t)\right)$. Setting $\omega(t) \equiv \mu(t)$ 


$$
\begin{aligned}
L_{2}(x ; \rho)= & \frac{1}{2}\left(\sum_{j \in N} \alpha_{j}\left(P_{j}^{g}\right)^{2}+\sum_{j \in N} \beta_{j}\left(P_{j}^{l}\right)^{2}+\sum_{j \in N} D_{j} \omega_{j}^{2}+\sum_{j} z_{j}^{2}\right)+\sum_{j \in N} \mu_{j}\left(P_{j}^{g}-P_{j}^{l}-p_{j}-D_{j} \omega_{j}+\sum_{i: i \rightarrow j} B_{i j} \theta_{i j}-\sum_{k: j \rightarrow k} B_{j k} \theta_{j k}\right) \\
& +\sum_{j \in N} \lambda_{j}\left(P_{j}^{g}-P_{j}^{l}-p_{j}+\sum_{i: i \rightarrow j} B_{i j} \phi_{i j}-\sum_{k: j \rightarrow k} B_{j k} \phi_{j k}\right)+\sum_{(i, j) \in E} \eta_{i j}^{-}\left(\underline{\theta}_{i j}-\phi_{i j}(t)\right)+\sum_{(i, j) \in E} \eta_{i j}^{+}\left(\phi_{i j}(t)-\bar{\theta}_{i j}\right)
\end{aligned}
$$

is equivalent to the primal update (8d) on $\omega(t)$, as in the pernode balance case. Moreover the control laws (6e)(6f) are then equivalent to

$$
\begin{aligned}
T^{g} \dot{P}^{g} & =\left[P^{g}(t)-\Gamma^{g} \nabla_{P^{g}} L_{2}(x(t), \rho(t))\right]_{\underline{P}^{g}}^{\bar{P}^{g}}-P^{g}(t) \\
T^{l} \dot{P}^{l} & =\left[P^{l}(t)-\Gamma^{l} \nabla_{P^{l}} L_{2}(x(t), \rho(t))\right]_{\underline{P}^{l}}^{\bar{P}^{l}}-P^{l}(t)
\end{aligned}
$$

i.e., the generator and controllable load at each node $j$ carry out the primal update $(8 \mathrm{a})$. For $(\tilde{\theta}, \tilde{\phi}),(1 \mathrm{a})$ and $(6 \mathrm{~d})$ are equivalent to the primal update $(8 \mathrm{a})$ :

$$
\begin{aligned}
\dot{\tilde{\theta}} & =-B^{-1} \nabla_{\tilde{\theta}} L_{2}(x(t), \rho(t)) \\
\dot{\tilde{\phi}} & =-\Gamma^{\tilde{\phi}} \nabla_{\tilde{\phi}} L_{2}(x(t), \rho(t))
\end{aligned}
$$

where $\Gamma^{\tilde{\phi}}:=\operatorname{diag}\left(\gamma_{i j}^{\tilde{\phi}},(i, j) \in E\right)$.

\section{Optimality of equilibrium point}

In this subsection, we address the optimality of the equilibrium point of the closed-loop system (1)(6). Given an $(x, \rho):=\left(\left(\tilde{\theta}, \tilde{\phi}, \omega, P^{g}, P^{l}\right),(\lambda, \mu),\left(\eta^{-}, \eta^{+}\right)\right)$, recall that the control input $u\left(x, \rho_{1}, \rho_{2}\right)$ is given by (7).

Definition 1. A point $\left(x^{*}, \rho^{*}\right):=\left(\tilde{\theta}^{*}, \tilde{\phi}^{*}, \omega^{*}, P^{g *}, P^{l *}, \lambda^{*}, \eta^{+*}\right.$, $\left.\eta^{-*}, \mu^{*}\right)$ is an equilibrium point or an equilibrium of the closed-loop system (1)(6) if

1) The right-hand side of (1) vanishes at $x^{*}$ and $u\left(x^{*}, \rho^{*}\right)$.

2) The right-hand side of (6a)-(6d) vanishes at $\left(x^{*}, \rho^{*}\right)$.

Definition 2. A point $\left(x^{*}, \rho^{*}\right)$ is primal-dual optimal if $\left(x^{*}, u\left(x^{*}, \rho^{*}\right)\right)$ is optimal for (5) and $\rho^{*}$ is optimal for its dual problem.

We make the following assumption:

A2: The problem (5) is feasible.

The following theorem characterizes the correspondence between the equilibrium of the closed-loop system (1)(6) and the primal-dual optimal solution of (5).

Theorem 1. Suppose A2 holds. A point $\left(x^{*}, \rho^{*}\right)$ is primal-dual optimal if and only if $\left(x^{*}, \rho^{*}\right)$ is an equilibrium of closed-loop system (1)(6) satisfying (2) and $\mu^{*}=0$.

Next result says that, at equilibrium, the network balance condition (4) and line limits (3) are satisfied and the nominal frequency is restored. Moreover the equilibrium is unique.

Theorem 2. Suppose A1 and A2 hold. Let $\left(x^{*}, \rho^{*}\right)$ be primaldual optimal. Then

1) The equilibrium $\left(x^{*}, \mu^{*}\right)$ is unique, with $\left(\theta^{*}, \phi^{*}\right)$ being unique up to (equilibrium) reference angles $\left(\theta_{0}, \phi_{0}\right)$.
2) The nominal frequency is restored, i.e., $\omega_{j}^{*}=0$ for all $j \in N$; moreover $\tilde{\phi}_{i j}^{*}=\tilde{\theta}_{i j}^{*}$ for all $(i, j) \in E$.

3) The network balance condition (4) is satisfied by $x^{*}$.

4) The line limits (3) are satisfied by $x^{*}$, implying $\underline{P}_{i j} \leq$ $P_{i j} \leq \bar{P}_{i j}$ on every tie line $(i, j) \in E$.

Theorem 2 shows that the equilibrium point has a simple yet intuitive structure. Moreover, Theorem 2 implies that the closed-loop system can autonomously eliminate congestions on tie lines. This feature has important implications. It means our distributed frequency control is capable of serving as a corrective re-dispatch without the coordination of dispatch centers if a congestion arises. This can enlarge the feasible region for economic dispatch, since corrective re-dispatch has been naturally taken into account.

The proofs of Theorem 1 and 2 are given in Appendix A.

\section{E. Asymptotic stability}

In this subsection, we address the asymptotic stability of the closed-loop system (1)(6), under an additional assumption:

A3: The initial state of the closed-loop system (1)(6) is finite, and $p_{j}^{g}(0), p_{j}^{l}(0)$ satisfy constraint (2).

As in the per-node balance case the closed-loop system (1)(6) satisfies constraint (2) even during transient.

Lemma 3. Suppose A1 and A3 hold. Then constraint (2) is satisfied for all $t>0$, i.e. $\left(P^{g}(t), P^{l}(t)\right) \in X$ for all $t \geq 0$ where $X$ is defined in (9).

The proof is exactly the same as that for Lemma 3 in [1] and omitted.

Similar to the per-node balance case, we first rewrite the closed-loop system using states $\tilde{\theta}, \tilde{\phi}$ instead of $\theta, \phi$ (they are equivalent under assumption A1). Setting $\mu \equiv \omega$, the closedloop system (1)(6) is equivalent to (in vector form):

$$
\begin{aligned}
\dot{\tilde{\theta}}(t) & =C^{T} \omega(t) \\
\dot{\omega}(t) & =M^{-1}\left(P^{g}(t)-P^{l}(t)-p-D \omega(t)-C B \tilde{\theta}(t)\right) \\
\dot{P}^{g}(t) & =\left(T^{g}\right)^{-1}\left(-P^{g}(t)+\hat{u}^{g}(t)\right) \\
\dot{P}^{l}(t) & =\left(T^{l}\right)^{-1}\left(-P^{l}(t)+\hat{u}^{l}(t)\right) \\
\dot{\eta}^{+}(t) & =\Gamma^{\eta}[\tilde{\phi}(t)-\bar{\theta}]_{\eta^{+}}^{+} \\
\dot{\eta}^{-}(t) & =\Gamma^{\eta}[\underline{\theta}-\tilde{\phi}(t)]_{\eta^{-}}^{+} \\
\dot{\lambda}(t) & =\Gamma^{\lambda}\left(P^{g}(t)-P^{l}(t)-p-C B \tilde{\phi}(t)\right) \\
\dot{\tilde{\phi}}(t) & =\Gamma^{\tilde{\phi}}\left(B C^{T} \lambda(t)+B C^{T} z(t)+\eta^{-}(t)-\eta^{+}(t)\right)(13 \mathrm{~h})
\end{aligned}
$$


where $z(t):=P^{g}(t)-P^{l}(t)-p-C B \tilde{\phi}(t)$ and

$$
\begin{aligned}
& \hat{u}^{g}(t)=\left[P^{g}(t)-\Gamma^{g}\left(A^{g} P_{j}^{g}(t)+\omega(t)+z(t)+\lambda(t)\right)\right]_{\underline{P}^{g}}^{\bar{P}^{g}} \\
& \hat{u}_{j}^{l}(t)=\left[P^{l}(t)-\Gamma^{l}\left(A^{l} P_{j}^{l}(t)-\omega(t)-z(t)-\lambda(t)\right)\right]_{\underline{P}^{l}}^{\bar{P}^{l}}
\end{aligned}
$$

Denote $w:=\left(\tilde{\theta}, \omega, P^{g}, P^{l}, \lambda, \eta^{+}, \eta^{-}, \tilde{\phi}\right)$.

Note that the right-hand sides of $(13 \mathrm{e})(13 \mathrm{f})$ are discontinuous due to projection to the nonnegative quadrant for $\left(\eta^{+}(t), \eta^{-}(t)\right)$. The system (13) is called a projected dynamical system and we adopt the concept of Caratheodory solutions for such a system where a trajectory $(w(t), t \geq 0)$ is called a Caratheodory solution, or just a solution, to (13) if it is absolutely continuous in $t$ and satisfies (13) almost everywhere. The result in [10, Theorems 2 and 3] implies that, given any initial state, there exists a unique solution trajectory to the closed-loop system (13) as the unprojected system is Lipschitz and the nonnegative quadrant is closed and convex. See [11, Theorem 3.1] for extension of this result to the Hilbert space.

With regard to system (13), we first define two sets, $\sigma^{+}$and $\sigma^{-}$, as follows [8].

$$
\begin{aligned}
& \sigma^{+}:=\left\{(i, j) \in E \mid \eta_{i j}^{+}=0, \tilde{\phi}_{i j}-\bar{\theta}_{i j}<0\right\} \\
& \sigma^{-}:=\left\{(i, j) \in E \mid \eta_{i j}^{-}=0, \underline{\theta}_{i j}-\tilde{\phi}_{i j}<0\right\}
\end{aligned}
$$

Then (6b) and (6c) are equivalent to

$$
\begin{aligned}
& \dot{\eta}_{i j}^{+}= \begin{cases}\gamma_{i j}^{\eta}\left(\tilde{\phi}_{i j}-\bar{\theta}_{i j}\right), & \text { if }(i, j) \notin \sigma^{+} ; \\
0, & \text { if }(i, j) \in \sigma^{+} .\end{cases} \\
& \dot{\eta}_{i j}^{-}= \begin{cases}\gamma_{i j}^{\eta}\left(\underline{\theta}_{i j}-\tilde{\phi}_{i j}\right), & \text { if }(i, j) \notin \sigma^{-} \\
0, & \text { if }(i, j) \in \sigma^{-} .\end{cases}
\end{aligned}
$$

In a fixed $\sigma^{+}, \sigma^{-}$, define $F(w)$.

$$
F(w)=\left[\begin{array}{l}
-B^{1 / 2} C^{T} \omega \\
-M^{-1 / 2}\left(P^{g}-P^{l}-p-D \omega-C B \tilde{\theta}\right) \\
\left(T^{g}\right)^{-1}\left(A^{g} P^{g}+\omega+z+\lambda\right) \\
\left(T^{l}\right)^{-1}\left(A^{l} P^{l}-\omega-z-\lambda\right) \\
-\left(\Gamma^{\eta}\right)^{1 / 2}[\tilde{\phi}-\bar{\theta}]_{\eta^{+}}^{+} \\
-\left(\Gamma^{\eta}\right)^{1 / 2}[\underline{\theta}-\tilde{\phi}]_{\eta^{-}}^{+} \\
-\left(\Gamma^{\lambda}\right)^{1 / 2}\left(P^{g}-P^{l}-p-C B \tilde{\phi}\right) \\
-\left(\Gamma^{\tilde{\phi}}\right)^{1 / 2}\left(B C^{T} \lambda+B C^{T} z+\eta^{-}-\eta^{+}\right)
\end{array}\right]
$$

If $\sigma^{+}$and $\sigma^{-}$do not change, $F(w)$ is continuously differentiable in $w$.

Similarly, we define $S:=\mathbb{R}^{m+n+1} \times X \times \mathbb{R}^{2 m+n+1+m}$, where the closed convex set $X$ is defined in (9). Then for any $w$ we define the projection of $w-F(w)$ onto $S$ as

$$
H(w):=\operatorname{Proj}_{S}(w-F(w)):=\arg \min _{y \in S}\|y-(w-F(w))\|_{2}
$$

Then the closed-loop system (13) is equivalent to

$$
\dot{w}(t)=\Gamma_{2}(H(w(t))-w(t))
$$

where the positive definite gain matrix is :

$$
\begin{array}{r}
\Gamma_{2}=\operatorname{diag}\left(B^{-1 / 2}, M^{-1 / 2},\left(T^{g}\right)^{-1},\left(T^{l}\right)^{-1},\right. \\
\left.\left(\Gamma^{\lambda}\right)^{1 / 2},\left(\Gamma^{\eta}\right)^{1 / 2},\left(\Gamma^{\eta}\right)^{1 / 2},\left(\Gamma^{\tilde{\phi}}\right)^{1 / 2}\right)
\end{array}
$$

Note that the projection operation $H$ has an effect only on $\left(P^{g} ; P^{l}\right)$ and Lemma 3 indicates that $w(t) \in S$ for all $t>0$, justifying the equivalence of (13) and (16).

A point $w^{*} \in S$ is an equilibrium of the closed-loop system (16) if and only if it is a fixed point of the projection $H\left(w^{*}\right)=$ $w^{*}$. Let $E_{2}:=\{w \in S \mid H(w(t))-w(t)=0\}$ be the set of equilibrium points. Then we have the following theorem.

Theorem 4. Suppose A1, A 2 and A 3 hold. Starting from any initial point $w(0), w(t)$ remains in a bounded set for all $t$ and $w(t) \rightarrow w^{*}$ as $t \rightarrow \infty$ for some equilibrium $w^{*} \in E_{2}$ that is optimal for problem (5).

For any equilibrium point $w^{*}$, we define the following function taking the same form as the per-node case.

$$
\begin{aligned}
\tilde{V}_{2}(w)= & -(H(w)-w)^{T} F(w)-\frac{1}{2}\|H(w)-w\|_{2}^{2} \\
& +\frac{1}{2} k\left(w-w^{*}\right)^{T} \Gamma_{2}^{-2}\left(w-w^{*}\right)
\end{aligned}
$$

where $k$ is small enough such that $\Gamma_{2}-k \Gamma_{2}^{-1}>0$ is strictly positive definite.

For any fixed $\sigma^{+}$and $\sigma^{-}, \tilde{V}_{2}$ is continuously differentiable as $F(w)$ is continuously differentiable in this situation. Similar to $V_{1}(w)$ used in Part I of the paper, we know $\tilde{V}_{2}(w) \geq 0$ on $S$ and $\tilde{V}_{2}(w)=0$ holds only at any equilibrium $w^{*}=H\left(w^{*}\right)$ [12]. Moreover, $\tilde{V}_{2}$ is nonincreasing for fixed $\sigma^{+}$and $\sigma^{-}$, as we prove in Appendix B.

It is worth to note that the index sets $\sigma^{+}$and $\sigma^{-}$may change sometimes, resulting in discontinuity of $\tilde{V}_{2}(w)$. To circumvent such an issue, we slightly modify the definition of $V_{2}(w)$ at the discontinuous points as:

1) $V_{2}(w):=\tilde{V}_{2}(w)$, if $\tilde{V}_{2}(w)$ is continuous at $w$;

2) $V_{2}(w):=\limsup _{v \rightarrow w} \tilde{V}_{2}(v)$, if $\tilde{V}_{2}(w)$ is discontinuous at $w$.

Then $V_{2}(w)$ is upper semi-continuous in $w$, and $V_{2}(w) \geq 0$ on $S$ and $V_{2}(w)=0$ holds only at any equilibrium $w^{*}=H\left(w^{*}\right)$. As $V_{2}(w)$ is not differentiable for $w$ at discontinuous points, we use the Clarke gradient as the gradient at these points [13].

Note that $\tilde{V}_{2}$ is continuous almost everywhere except the switching points. Hence both $V_{2}(w)$ is nonpathological [14], [15]. With these definitions and notations above, we can prove Theorem 4. The detail of proof is provided in Appendix B.

\section{CASE STUDIES}

\section{A. System configuration}

A four-area system based on Kundur's four-machine, twoarea system [16] [17] is used to test our optimal frequency controller. There are one (aggregate) generator (Gen1 Gen4), one controllable (aggregate) load (L1c $\sim 44$ c) and one uncontrollable (aggregate) load (L1 L4) in each area, which is shown in Fig.1. The parameters of generators and controllable loads are given in Table I. The total uncontrollable load in each area are identically $480 \mathrm{MW}$. At time $t=10 \mathrm{~s}$, we add step changes on the uncontrollable loads in four areas to test the performance of our controllers.

All the simulations are implemented in PSCAD [18] with 8GB memory and $2.39 \mathrm{GHz}$ CPU. We use the detailed electromagnetic transient model of three-phase synchronous 
machines to simulate generators with both governors and exciters. The uncontrollable load L1-L4 are modelled by the fixed load in PSCAD, while controllable load L1c-L4c are formulated by the self-defined controlled current source. The closed-loop system diagram is shown in Fig.2. We need measure loacal frequency, generation, controllable load and tie-line power flows to compute control demands. Only $\tilde{\phi}_{i j}$ are exchanged between neighbors. All variables are added by their initial steady state values to explicitly show the actual values.

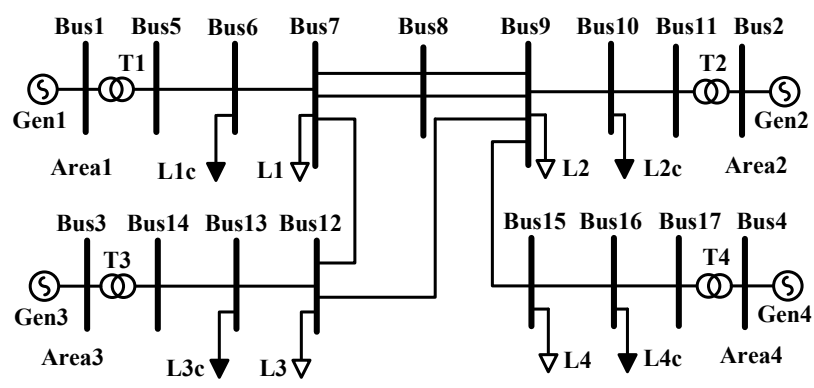

Fig. 1: Four-area power system

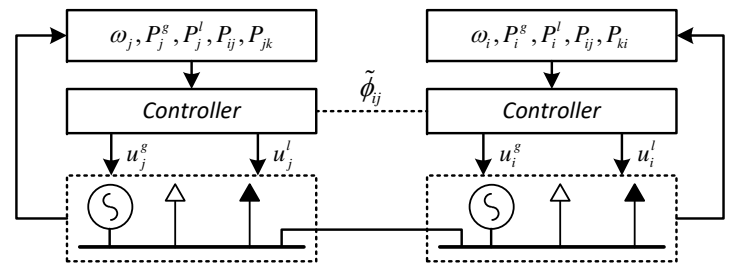

Fig. 2: Closed-loop system diagram

TABLE I:

SYSTEM PARAMETERS

\begin{tabular}{ccccccc}
\hline Area $j$ & $D_{j}$ & $R_{j}$ & $\alpha_{j}$ & $\beta_{j}$ & $T_{j}^{g}$ & $T_{j}^{l}$ \\
\hline 1 & 0.04 & 0.04 & 2 & 2.5 & 4 & 4 \\
2 & 0.045 & 0.06 & 2.5 & 4 & 6 & 5 \\
3 & 0.05 & 0.05 & 1.5 & 2.5 & 5 & 4 \\
4 & 0.055 & 0.045 & 3 & 3 & 5.5 & 5 \\
\hline
\end{tabular}

\section{B. Network Power Balance Case}

In this case, the generations in each area are initiated as (560.9, 548.7, 581.2, 540.6) MW and the controllable loads $(70.8,89.6,71.3,79.4) \mathrm{MW}$. The load changes are identical to those in Table II, which are also unknown to the controllers. We use method in Remark 4 to estimate the load changes. Operational constraints on generations, controllable loads and tie lines are shown in Table II.

TABLE II:

\section{CAPACITY LIMITS IN NETWORK CASE}

\begin{tabular}{ccccc}
\hline & Area 1 & Area 2 & Area 3 & Area 4 \\
\hline$\left[\underline{P}_{j}^{g}, \bar{P}_{j}^{g}\right](\mathrm{MW})$ & {$[550,710]$} & {$[530,680]$} & {$[550,700]$} & {$[530,670]$} \\
\hline$\left[\underline{P}_{j}^{l}, \bar{P}_{j}^{l}\right](\mathrm{MW})$ & {$[20,80]$} & {$[60,100]$} & {$[20,80]$} & {$[35,80]$} \\
\hline \hline Tie line & $(2,1)$ & $(3,1)$ & $(3,2)$ & $(4,2)$ \\
\hline$\left[\underline{P}_{i j}, \bar{P}_{i j}\right](\mathrm{MW})$ & {$[-65,65]$} & {$[-65,65]$} & {$[-65,65]$} & {$[-65,65]$} \\
\hline
\end{tabular}

1) Stability and optimality: The dynamics of local frequencies and tie-line power flows are illustrated in Fig.3. The frequencies are well restored in all four control areas while the tie line powers are remained within their acceptable ranges. The generations and controllable loads are different from that before disturbance, indicating that the system is stabilized at a new steady state. The resulting equilibrium point is given in Table IV, which is identical to the optimal solution of (5) computed by centralized optimization using CVX. These simulation results confirm that our controller can autonomously guarantee the frequency stability while achieving optimal operating point in the overall system.
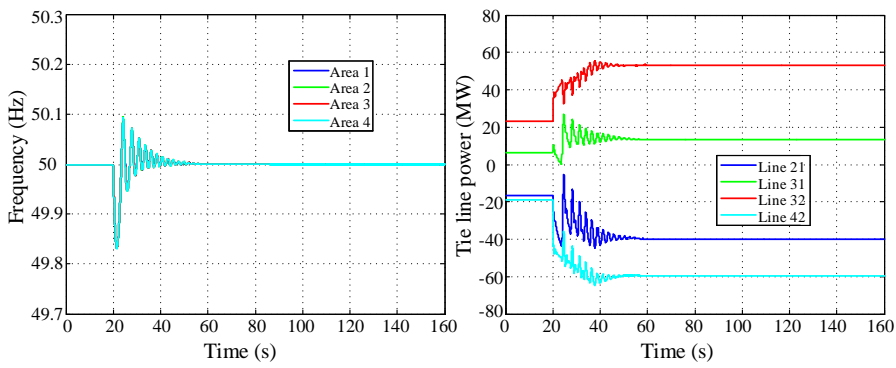

Fig. 3: Dynamics of frequency (left) and tie-line flows (right) in network balance case

TABLE III: EQUILIBRIUM POINTS

\begin{tabular}{ccccc}
\hline & Area 1 & Area 2 & Area 3 & Area 4 \\
\hline$P_{j}^{g *}(\mathrm{MW})$ & 620 & 596 & 660 & 580 \\
$P_{j}^{l *}(\mathrm{MW})$ & 23.6 & 59.8 & 23.6 & 39.7 \\
\hline \hline Tie line & $(2,1)$ & $(3,1)$ & $(3,2)$ & $(4,2)$ \\
\hline$P_{i j}^{*}(\mathrm{MW})$ & -39.94 & 13.35 & 53.27 & -59.6 \\
\hline
\end{tabular}

2) Dynamic performance: In this subsection, we analyze the impacts of operational (capacity and line power) constraints on the dynamic property. Similarly, we compare the responses of frequency controllers with and without considering input saturations. The trajectories of mechanical power of turbines and controllable loads are shown in Fig.4 and Fig.5, respectively. In this case, the system frequency is restored, and the same optimal equilibrium point is achieved.

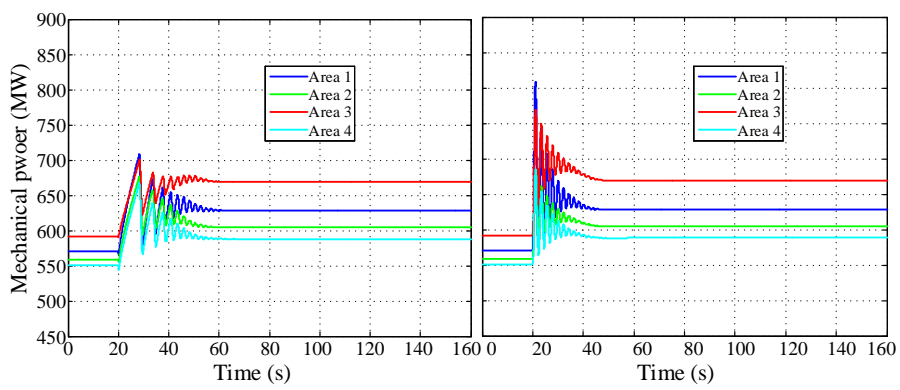

Fig. 4: Mechanical outputs with(left)/without(right) capacity constraints

3) Congestion analysis: In this scenario, we reduce tieline power constraints to $\bar{P}_{i j}=-\underline{P}_{i j}=50 \mathrm{MW}$, which causes congestions in tie-line $(2,3)$ and $(2,4)$. The steady states 


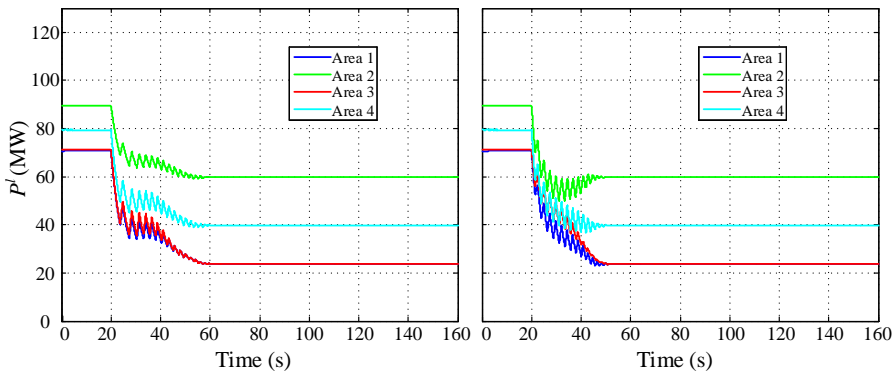

Fig. 5: Controllable loads with(left)/without(right) capacity constraints

under the distributed control are listed in Table IV. Note that $P_{j}^{g *}-p_{j}+P_{j}^{l *}-\sum_{k: j \rightarrow k} P_{j k}^{*}+\sum_{i: i \rightarrow j} P_{i j}^{*}=0$ hold in each area.

The dynamics of tie-line powers in two different scenarios shown in Fig.6 indicate that $(2,4)$ reaches the limit in steady state. However, by adopting the proposed fully distributed optimal frequency control, the congestion is eliminated and all the tie line powers remain within the limits. Thus, congestion control is achieved optimally in a distributed manner.

TABLE IV:

Simulation Results With CONGESTION

\begin{tabular}{ccccc}
\hline & Area 1 & Area 2 & Area 3 & Area 4 \\
\hline$P_{j}^{g *}(\mathrm{MW})$ & 618 & 595 & 658 & 585 \\
$P_{j}^{l *}(\mathrm{MW})$ & 25.1 & 60.7 & 25.1 & 34.9 \\
\hline \hline Tie line & $(2,1)$ & $(3,1)$ & $(3,2)$ & $(4,2)$ \\
\hline$P_{i j}^{*}(\mathrm{MW})$ & -36.4 & 13.1 & 49.5 & -49.9 \\
\hline
\end{tabular}

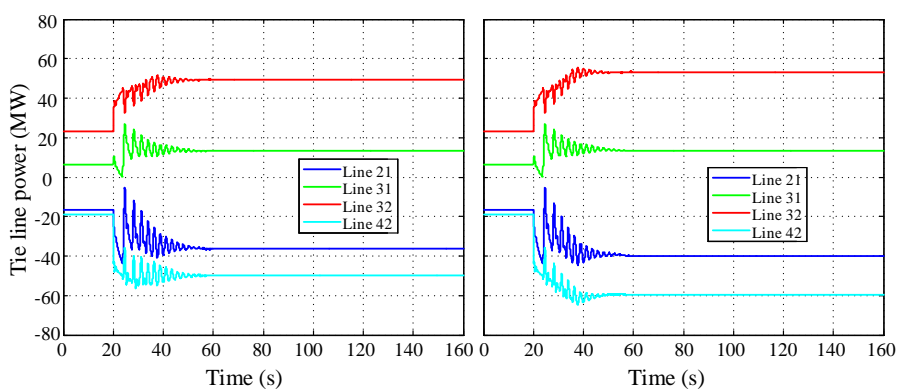

Fig. 6: Tie line power with(left)/without(right) capacity constraints

\section{CONCLUding REMARKS}

In this paper, we have devised a distributed optimal frequency control in the network balance case, which can autonomously restore the nominal frequencies after unknown load disturbances while minimizing the regulation costs. The capacity constraints on the generations and controllable loads can also be satisfied even during transient. In addition, congestions can be eliminated automatically, implying tie-line powers can be remained within given ranges. Only neighborhood communication is required in this case. Like the per-node case, here the closed-loop system again carries out a primaldual algorithm with saturation to solve the associated optimal problem. To cope with the discontinuity introduced due to enforcing different types of constraints, we have constructed a nonpathological Lyapunov function to prove the asymptotically stability of the closed-loop systems. Simulations on a modified Kundur's power system validate the effectiveness of our controller.

This approach is also applicable to other problem involving frequency regulation, e.g. standalone microgrid or demand side management. We highlight two crucial implications of our work: First, our distributed frequency control is capable of serving as an automatically corrective re-dispatch without the coordination of dispatch center when certain congestion happens; Second, the feasible region of economic decisions can be enlarged benefiting from the corrective re-dispatch. In this sense, our work may provide a systematic way to bridge the gap between the (secondary) frequency control in a fast timescale and the economic dispatch in a slow timescale, hence breaking the traditional hierarchy of the power system frequency control and economic dispatch.

\section{REFERENCES}

[1] Z. Wang, F. Liu, S. H. Low, C. Zhao, and S. Mei, "Distributed frequency control with operational constraints, part i: Per-node power balance," https://arxiv.org/abs/1702.07965, 2017.

[2] E. Mallada, C. Zhao, and S. H. Low, "Optimal load-side control for frequency regulation in smart grids," IEEE Trans. Autom. Control, to appear, 2017.

[3] T. Stegink, C. D. Persis, and A. van der Schaft, "A unifying energybased approach to stability of power grids with market dynamics," arXiv preprint arXiv:1604.05200, 2016.

[4] T. Stegink, C. D. Persis, and A. van der Schaft, "A port-hamiltonian approach to optimal frequency regulation in power grids," in Proc. 54th IEEE Conference on Decision and Control (CDC), Dec 2015, pp. 32243229.

[5] P. Yi, Y. Hong, and F. Liu, "Distributed gradient algorithm for constrained optimization with application to load sharing in power systems," Syst. Control Lett., vol. 83, pp. 45-52, Sept 2015.

[6] C. Zhao, E. Mallada, S. Low, and J. Bialek, "A unified framework for frequency control and congestion management," in Power Systems Computation Conference (PSCC), 2016. IEEE, 2016, pp. 1-7.

[7] X. Zhang and A. Papachristodoulou, "A real-time control framework for smart power networks: Design methodology and stability," Automatica, vol. 58, pp. 43-50, 2015.

[8] D. Feijer and F. Paganini., "Stability of primal-dual gradient dynamics and applications to network optimization," Automatica, vol. 46, no. 12, pp. 1974-1981, Dec 2010.

[9] A. Cherukuri, E. Mallada, and J. Corts, "Asymptotic convergence of constrained primaldual dynamics," Syst. Control Lett., vol. 87, pp. $10-$ 15, 2016.

[10] P. Dupuis and A. Nagurney, "Dynamical systems and variational inequalities," Annals of Operations Research, vol. 44, pp. 9-42, 1993.

[11] M.-G. Cojocaru and L. B. Jonker, "Existence of solutions to projected differential equations in hilbert spaces," Proc. Amer. Math. Soc., vol. 132, pp. 183-193, 2004.

[12] M. Fukushima, "Equivalent differentiable optimization problems and descent methods for asymmetric variational inequality problems," Math. programming, vol. 53, no. 1-3, pp. 99-110, Jan 1992.

[13] F. H. Clarke, Optimization and nonsmooth analysis. Siam, 1990, vol. 5.

[14] A. Bacciotti and F. Ceragioli, "Nonpathological lyapunov functions and discontinuous carathodory systems," Automatica, vol. 42, no. 3, pp. 453 $-458,2006$.

[15] A. Bacciotti and F. Ceragioli, "Stability and stabilization of discontinuous systems and nonsmooth lyapunov functions," ESAIM: Control, Optimisation and Calculus of Variations, vol. 4, pp. 361-376, 1999.

[16] J. Fang, W. Yao, Z. Chen, J. Wen, and S. Cheng, "Design of anti-windup compensator for energy storage-based damping controller to enhance power system stability," IEEE Trans. Power Syst., vol. 29, no. 3, pp. 1175-1185, May 2014.

[17] P. Kundur, Power System Stability and Control. McGraw-hill New York, 1994, vol. 7.

[18] https://hvdc.ca/pscad/. 


\section{APPENDIX A}

\section{PROOFS OF THEOREM 1 AND THEOREM 2}

We start with a lemma.

Lemma A.1. Suppose $\left(x^{*}, u^{*}\right)$ is optimal for (5). Then

1) $\omega^{*}=0$, i.e., the nominal frequency is restored;

2) the network balance condition (4) is satisfied by $x^{*}$;

3) $\phi^{*}-\theta^{*}=\left(\phi_{0}^{*}-\theta_{0}^{*}\right) \mathbf{1}$;

4) $\underline{\theta}_{i j} \leq \theta_{i j}^{*} \leq \bar{\theta}_{i j}$, i.e., the line limits (3) are satisfied.

Proof. Suppose $\left(x^{*}, u^{*}\right)$ is optimal but $\omega^{*} \neq 0$. Then $(5 \mathrm{c})$ implies

$$
P^{g *}-P^{l *}-p=C B C^{T} \phi^{*}
$$

Consider $\hat{x}:=\left(\hat{\theta}, \phi^{*}, \hat{\omega}, P^{g *}, P^{l *}\right)$ with $\hat{\theta}:=\phi^{*}$ and $\hat{\omega}:=0$. Then $\hat{x}$ satisfies $(5 \mathrm{~b})(5 \mathrm{c})$ due to (A.1). Hence $\left(\hat{x}, u^{*}\right)$ is feasible for (5) but has a strictly lower cost, contradicting the optimality of $\left(x^{*}, u^{*}\right)$. Hence $\omega^{*}=0$.

Multiplier both sides of (A.1) by $\mathbf{1}^{T}$ yields the network balance condition (4), proving 2 ).

To prove 3$)$, setting $\omega^{*}=0$ in (5b) and combining with (5c) yield

$$
C B C^{T} \theta^{*}=P^{g *}-P^{l *}-p=C B C^{T} \phi^{*}
$$

Since $C B C^{T}$ is an $(n+1) \times(n+1)$ matrix with rank $n$, its null space has dimension 1 and is spanned by $\mathbf{1}$ because $C^{T} \mathbf{1}=0$. Hence $C B C^{T}\left(\phi^{*}-\theta^{*}\right)=0$ implies that $\phi^{*}-\theta^{*}=\left(\phi_{0}^{*}-\theta_{0}^{*}\right) \mathbf{1}$. To prove 4), note that $\tilde{\phi}=C^{T} \phi$ and $\tilde{\theta}=C^{T} \theta$ and hence

$$
\tilde{\phi}^{*}-\tilde{\theta}^{*}=C^{T}\left(\phi^{*}-\theta^{*}\right)=\left(\phi_{0}^{*}-\theta_{0}^{*}\right) C^{T} \mathbf{1}=0
$$

i.e. $\tilde{\phi}^{*}=\tilde{\theta}^{*}$. We conclude from (5d) that $\underline{\theta}_{i j} \leq \theta_{i j}^{*} \leq \bar{\theta}_{i j}$. This completes the proof.

We have the following result.

Lemma A.2. Suppose $\left(x^{*}, \rho^{*}\right)$ is primal-dual optimal. Then

$$
\begin{aligned}
u_{j}^{g *}=P_{j}^{g *} & =\left[P_{j}^{g *}-\gamma_{j}^{g}\left(\alpha_{j} P_{j}^{g *}+\omega_{j}^{*}+z_{j}^{*}+\lambda_{j}^{*}\right)\right]_{\underline{P}_{j}^{g}}^{\bar{P}_{j}^{g}} \\
u_{j}^{l *}=P_{j}^{l *} & =\left[P_{j}^{l *}-\gamma_{j}^{l}\left(\beta_{j} P_{j}^{l *}-\omega_{j}^{*}-z_{j}^{*}-\lambda_{j}^{*}\right)\right]_{\underline{P}_{j}^{l}}^{\bar{P}_{j}^{l}}
\end{aligned}
$$

for any $\gamma_{j}^{g}>0$ and $\gamma_{j}^{l}>0$.

Lemma A.2 shows that the saturation of control input does not impact the optimal solution of optimization problem (5).

With Lemma 3, Lemma A.1 and Lemma A.2, we now can prove Theorem 1 and Theorem 2 .

Proof of Theorem 1. $\Rightarrow$ : Suppose $\left(x^{*}, \rho^{*}\right)$ is primal-dual optimal. Then $x^{*}$ satisfies the operational constraints (2). Moreover the right-hand side of (1) vanishes because:

- $\dot{\theta}=0$ since $\omega^{*}=0$ from Lemma A.1.

- $\dot{\omega}=0$ since constraint (5b) holds for $x^{*}$.

- $\dot{P}^{g}=\dot{P}^{l}=0$ since $\omega^{*}=0$ and $x^{*}$ satisfies (5e) and (5f).

- $\dot{\lambda}=0$ since (5c) holds for $x^{*}$.

- $\dot{\eta}^{+}=\dot{\eta}^{-}=0$ since (5d) holds for $x^{*}$.

- From (12f) we have

$$
\dot{\tilde{\phi}}=-\Gamma^{\tilde{\phi}} \nabla_{\tilde{\phi}} L_{2}\left(x^{*}, \rho^{*}\right)
$$

Since $\left(x^{*}, \rho^{*}\right)$ is a saddle point, we must have $\nabla_{\tilde{\phi}} L_{2}\left(x^{*}, \rho^{*}\right)=0$, implying $\dot{\tilde{\phi}}=0$.

Hence $\left(x^{*}, \rho^{*}\right)$ is an equilibrium of the closed-loop system (1)(6) that satisfies the operational constraints (2). Moreover $\mu^{*}=\omega^{*}=0$ since $\frac{\partial L_{2}}{\partial \omega_{j}}\left(x^{*}, \rho^{*}\right)=D_{j}\left(\omega_{j}^{*}-\mu_{j}^{*}\right)=0$ and $D_{j}>0$ for all $j \in N$.

$\Leftarrow$ : Suppose now $\left(x^{*}, \rho^{*}\right)$ is an equilibrium of the closed-loop system (1)(6) that satisfies (2) and $\mu^{*}=0$. Since (5) is convex with linear constraints, $\left(x^{*}, \rho^{*}\right)$ is a primal-dual optimal if and only if $\left(x^{*}, u\left(x^{*}, \rho^{*}\right)\right)$ is primal feasible and satisfies

$$
x^{*}=\arg \min _{x}\left\{L_{2}\left(x ; \rho^{*}\right) \mid\left(x, u\left(x, \rho^{*}\right)\right) \text { satisfies(2),(5e),(5f) }\right\}
$$

This is because $\nabla_{\rho_{1}} L_{2}\left(x^{*}, \rho^{*}\right)=0$ since $\dot{\mu}=\dot{\lambda}=0, \eta^{+*} \geq$ $0, \eta^{-*} \geq 0$, and the complementary slackness condition $\eta_{i j}^{+*}\left(\tilde{\phi}_{i j}^{*}-\bar{\theta}_{i j}\right)=0, \eta_{i j}^{-*}\left(\underline{\theta}_{i j}-\tilde{\phi}_{i j}^{*}\right)=0$ is satisfied since $\dot{\eta}^{+}=$ $\dot{\eta}^{-}=0$.

To show that $\left(x^{*}, u\left(x^{*}, \rho^{*}\right)\right)$ is primal feasible, note that since $\left(x^{*}, u\left(x^{*}, \rho^{*}\right)\right)$ is an equilibrium of (1), it satisfies $\omega^{*}=0$ and hence $(5 \mathrm{e})(5 \mathrm{f})$, in addition to (2). Moreover $\dot{\omega}=0$ means $\left(x^{*}, u\left(x^{*}, \rho^{*}\right)\right)$ satisfies (5b), $\dot{\lambda}=0$ implies (5c), $\dot{\eta}^{+}=\dot{\eta}^{-}=0$ implies (5d).

To show that $\left(x^{*}, \rho^{*}\right)$ satisfies (A.2), note that $(5 e)(5 f)$ and (6e)(6f) imply that

$$
\begin{aligned}
& P_{j}^{g *}=\left[P_{j}^{g *}-\gamma_{j}^{g}\left(\alpha_{j} P_{j}^{g *}+\omega_{j}^{*}+z_{j}^{*}+\lambda_{j}^{*}\right)\right]_{\underline{P}_{j}^{g}}^{\bar{P}_{j}^{g}} \\
& P_{j}^{l *}=\left[P_{j}^{l *}-\gamma_{j}^{l}\left(\beta_{j} P_{j}^{l *}-\omega_{j}^{*}-z_{j}^{*}-\lambda_{j}^{*}\right)\right]_{\underline{P}_{j}^{l}}^{\bar{P}_{j}^{l}}
\end{aligned}
$$

The rest of the proof follows the same line of argument as that in Theorem 1 in [1]. This proves that $\left(x^{*}, \rho^{*}\right)$ is primal-dual optimal and completes the proof of Theorem 1 .

Next we prove Theorem 2 .

Proof of Theorem 2. Let $\left(x^{*}, \rho^{*}\right)=\left(\tilde{\theta}^{*}, \tilde{\phi}^{*}, \omega^{*}, P^{g *}, P^{l *}\right.$, $\left.\lambda^{*}, \eta^{+*}, \eta^{-*}, \mu^{*}\right)$ be primal-dual optimal. For the uniqueness of $x^{*},\left(\omega^{*}, P^{g *}, P^{l *}\right)$ are unique because the objective function in (5) is strictly convex in $\left(\omega, P^{g}, P^{l}\right)$. Hence $\mu^{*}=\omega^{*}$ is unique as well. Assumption A1 that $\phi_{0}^{*}:=0$ and (A.1) imply that $\phi^{*}$ is uniquely determined by the equilibrium $\left(\omega^{*}, P^{g *}, P^{l *}\right)$. Since $\theta^{*}-\phi^{*}=\left(\theta_{0}^{*}-\phi_{0}^{*}\right) \mathbf{1}$, assumption A1 that $\theta_{0}^{*}:=0$ then implies that $\theta^{*}$ is unique. This proves the uniqueness of $\left(x^{*}, \mu^{*}\right)$.

The remaining three parts of the theorem follow from Lemma A.1.

\section{APPENDIX B \\ Proof THEOREM 4}

We start with a lemma.

Lemma B.1. Suppose A1, A4 and A5 hold. Then

1) $\dot{V}_{2}(w(t)) \leq 0$ in a fixed $\sigma^{+}$and $\sigma^{-}$.

2) The trajectory $w(t)$ is bounded, i.e., there exists $\bar{w}$ such that $\|w(t)\| \leq \bar{w}$ for all $t \geq 0$. 


$$
Q=\left[\begin{array}{llllllll}
0 & -B C^{T} & 0 & 0 & 0 & 0 & 0 & 0 \\
C B & D & -I_{|N|} & I_{|N|} & 0 & 0 & 0 & 0 \\
0 & I_{|N|} & A^{g}+I_{|N|} & -I_{|N|} & 0 & 0 & 0 & -C B \\
0 & -I_{|N|} & -I_{|N|} & A^{l}+I_{|N|} & 0 & 0 & 0 & C B \\
0 & 0 & -I_{|N|} & I_{|N|} & 0 & 0 & 0 & C B \\
0 & 0 & 0 & 0 & 0 & 0 & 0 & -I_{|E|-\left|\sigma^{+}\right|} \\
0 & 0 & 0 & 0 & 0 & 0 & 0 & I_{|E|-\left|\sigma^{-}\right|} \\
0 & 0 & -B C^{T} & B C^{T} & -B C^{T} & I_{|E|-\left|\sigma^{+}\right|} & -I_{|E|-\left|\sigma^{-}\right|} & B C^{T} C B
\end{array}\right]
$$

Proof of Lemma B.1. Given fixed $\sigma^{+}, \sigma^{-}$, for all $(i, j) \notin \sigma^{+}$, $(i, j) \notin \sigma^{-}$, we have

$$
\begin{aligned}
\dot{V}_{2}(w) & \leq k\left(H(w)-w^{*}\right)^{T} \cdot \Gamma_{2}^{-1}(H(w)-(w-F(w))) \\
& -(H(w)-w)^{T} \Gamma_{2} \cdot Q \cdot \Gamma_{2}(H(w)-w) \\
& -(H(w)-(w-F(w)))^{T}\left(\Gamma_{2}-k \Gamma_{2}^{-1}\right)(w-H(w)) \\
& -k\left(w-w^{*}\right)^{T} \cdot \Gamma_{2}^{-1} F(w)
\end{aligned}
$$

where $Q$ is a semi-definite positive matrix and $\Gamma_{2} Q=\nabla_{w} F(w)$, which given in (B.2). Here, the subscript of $I$ means its dimension, and $|A|$ means the cardinality of set $A$.

Given fixed $\sigma^{+}$and $\sigma^{-}, F(w)$ is continuous differentiable. In this case, (B.1a) and (B.1c) are nonpositive due to as discussed in the proof of Theorem 4 in [1]. (B.1b) is also nonpositive as $Q$ is semi-definite positive (see Eq. (B.2)).

Next, we prove that (B.1d) is nonpositive. Similar to the per-node case, substituting $\mu(t) \equiv \omega(t)$ into the Lagrangian $L_{2}(x, \rho)$ in (11) we obtain a function $\hat{L}_{2}(w)$ defined as follows.

$$
\begin{aligned}
\hat{L}_{2}(w) & :=L_{2}\left(\tilde{\theta}, \omega, P^{g}, P^{l}, \lambda, \mu, \eta^{+}, \eta^{-}, \tilde{\phi}\right)_{\mu=\omega} \\
& =\frac{1}{2}\left(\left(P^{g}\right)^{T} A^{g} P^{g}+\left(P^{l}\right)^{T} A^{l} P^{l}-\omega^{T} D \omega+z^{T} z\right) \\
& +\lambda^{T}\left(P^{g}-P^{l}-p-C B \tilde{\phi}\right)+\omega^{T}\left(P^{g}-P^{l}-p-C B \tilde{\theta}\right) \\
& +\left(\eta^{-}\right)^{T}(\underline{\theta}-\tilde{\phi})+\left(\eta^{+}\right)^{T}(\tilde{\phi}-\bar{\theta})
\end{aligned}
$$

In addition, denote $w_{1}=\left(\tilde{\theta}, P^{g}, P^{l}, \tilde{\phi}\right), w_{2}=\left(\lambda, \omega, \eta^{+}, \eta^{-}\right)$. Then $\hat{L}_{2}$ is convex in $w_{1}$ and concave in $w_{2}$.

In $F(w),[\tilde{\phi}-\overline{\boldsymbol{\theta}}]_{\eta^{+}}^{+}$and $[\underline{\theta}-\tilde{\phi}]_{\eta^{-}}^{+}$have unknown dimensions (up to $\sigma^{+}$and $\sigma^{-}$). Fortunately, we have

$$
\begin{aligned}
\left(\eta^{+}-\eta^{+*}\right)^{T}[\tilde{\phi}-\bar{\theta}]_{\eta^{+}}^{+} & \leq\left(\eta^{+}-\eta^{+*}\right)^{T}(\tilde{\phi}-\bar{\theta}) \\
& =\left(\eta^{+}-\eta^{+*}\right)^{T} \nabla_{\eta^{+}} \hat{L}_{2}
\end{aligned}
$$

where the inequality holds since $\eta_{i j}^{+}=0 \leq \eta_{i j}^{+*}$ and $\tilde{\phi}_{i j}-\bar{\theta}_{i j}<$ 0 for $(i, j) \in \sigma^{+}$, i.e., $\left(\eta_{i j}^{+}-\eta_{i j}^{+*}\right) \cdot\left(\tilde{\phi}_{i j}-\bar{\theta}_{i j}\right) \geq 0$. Similarly,

$$
\begin{aligned}
\left(\eta^{-}-\eta^{-*}\right)^{T}[\underline{\theta}-\tilde{\phi}]_{\eta^{-}}^{+} & \leq\left(\eta^{-}-\eta^{-*}\right)^{T}(\underline{\theta}-\tilde{\phi}) \\
& =\left(\eta^{-}-\eta^{-*}\right)^{T} \nabla_{\eta^{-}} \hat{L}_{2} .
\end{aligned}
$$

Consequently, it can be verified that

$$
\begin{aligned}
\left(w-w^{*}\right)^{T} \Gamma_{2}^{-1} F(w) & \leq\left(w-w^{*}\right)^{T} \nabla_{w} \hat{L}_{2}(w) \\
& =\left(w-w^{*}\right)^{T}\left[\begin{array}{r}
\nabla_{w_{1}} \hat{L}_{2} \\
-\nabla_{w_{2}} \hat{L}_{2}
\end{array}\right]\left(w_{1}, w_{2}\right)
\end{aligned}
$$

where, $\nabla_{w_{1}} \hat{L}_{2}:=\left[\begin{array}{c}\nabla_{\tilde{\theta}} \hat{L}_{2} \\ \nabla_{P s} \hat{L}_{2} \\ \nabla_{P l} \hat{L}_{2} \\ \nabla_{\tilde{\phi}} \hat{L}_{2}\end{array}\right]$ and $\nabla_{w_{2}} \hat{L}_{2}:=\left[\begin{array}{c}\nabla_{\lambda} \hat{L}_{2} \\ \nabla_{\omega} \hat{L}_{2} \\ \nabla_{\eta^{+}}+\hat{L}_{2} \\ \nabla_{\eta^{-}}-\hat{L}_{2}\end{array}\right]$.

Then we have

$$
-k\left(w-w^{*}\right)^{T} \Gamma_{2}^{-1} F(w) \leq-k\left(w-w^{*}\right)^{T} \cdot \Gamma_{2}^{-1} F(w)
$$

$=-k\left(w_{1}-w_{1}^{*}\right)^{T} \nabla_{w_{1}} \hat{L}_{2}\left(w_{1}, w_{2}\right)+k\left(w_{2}-w_{2}^{*}\right)^{T} \nabla_{w_{2}} \hat{L}_{2}\left(w_{1}, w_{2}\right)$

$\leq k\left(\hat{L}_{2}\left(w_{1}^{*}, w_{2}\right)-\hat{L}_{2}\left(w_{1}, w_{2}\right)+\hat{L}_{2}\left(w_{1}, w_{2}\right)-\hat{L}_{2}\left(w_{1}, w_{2}^{*}\right)\right)$

$=k(\underbrace{\hat{L}_{2}\left(w_{1}^{*}, w_{2}\right)-\hat{L}_{2}\left(w_{1}^{*}, w_{2}^{*}\right)}_{\leq 0}+\underbrace{\hat{L}_{2}\left(w_{1}^{*}, w_{2}^{*}\right)-\hat{L}_{2}\left(w_{1}, w_{2}^{*}\right)}_{\leq 0})$

$\leq 0$

where the first inequality holds because $\hat{L}_{2}$ is convex in $w_{1}$ and concave in $w_{2}$ and the second inequality follows because $\left(w_{1}^{*}, w_{2}^{*}\right)$ is a saddle point. Therefore (B.1d) is nonpositive, proving the first assertion.

To prove the second assertion, we further investigate the situation that $\sigma^{+}$or $\sigma^{-}$changes. We only consider the set $\sigma^{+}$ since it is the same to $\sigma^{-}$. We have the following observations:

- The set $\sigma^{+}$is reduced, which only happens when $\tilde{\phi}_{i j}-\bar{\theta}_{i j}$ goes through zero, from negative to positive. Hence an extra term will be added to $V_{2}$. As this term is initially zero, there is no discontinuity of $V_{2}$ in this case.

- The set $\sigma^{+}$is enlarged when $\eta_{i j}^{+}$goes to zero from positive while $\tilde{\phi}_{i j}<\bar{\theta}_{i j}$. Here $V_{2}$ will lose a positive term $\left(\gamma_{i j}^{\eta}\right)^{2}\left(\tilde{\phi}_{i j}-\bar{\theta}_{i j}\right)^{2} / 2$, causing discontinuity.

In the context, we conclude that $V_{2}$ is always nonincreasing along the trajectory even when $\sigma^{+}$or $\sigma^{-}$changes and discontinuity occurs.

To prove that the trajectory $w(t)$ is bounded note that $[12$, Theorem 3.1] proves that $\hat{V}_{2}(w):=-(H(w)-w)^{T} F(w)-$ $\frac{1}{2}\|H(w)-w\|_{2}^{2}$ satisfies $\hat{V}_{2}(w) \geq 0$ over $S$. Therefore, we have

$$
\frac{1}{2} k\left(w(t)-w^{*}\right)^{T} \Gamma_{2}^{-2}\left(w(t)-w^{*}\right) \leq V_{2}(w(t)) \leq V_{2}(w(0))
$$

indicating the trajectory $w(t)$ is bounded.

Lemma B.2. Suppose A1, A4 and A5 hold. Then

1) The trajectory $w(t)$ converges to the largest weakly invariant subset $W_{2}^{*}$ contained in $W_{2}:=\left\{w \in S \mid \dot{V}_{2}(w)=0\right\}$.

2) Every point $w^{*} \in W_{2}^{*}$ is an equilibrium point of (16).

Proof of Lemma B.2. Given an initial point $w(0)$ there is a compact set $\Omega_{0}:=\Omega(w(0)) \subset S$ such that $w(t) \in \Omega_{0}$ for $t \geq 0$ and $\dot{V}_{2}(w) \leq 0$ in $\Omega_{0}$. 
Invoking the proof of Lemma B.1, $V_{2}$ is radially unbounded and positively definite except at equilibrium. As $V_{2}$ and $\dot{V}_{2}$ are nonpathological, we conclude that any trajectory $w(t)$ starting from $\Omega_{0}$ converges to the largest weakly invariant subset $W_{2}^{*}$ contained in $W_{2}=\left\{w \in \Omega_{0} \mid \dot{V}_{2}(w)=0\right\}$ [14, Proposition 3], proving the first assertion.

For the second assertion, We fix $w(0) \in W_{2}^{*}$ and then prove that $w(0)$ must be an equilibrium point.

From (B.1b), direct computing yields

$$
\begin{aligned}
\dot{V}_{2}(w(t)) & \leq-\dot{\omega}^{T} D \dot{\omega}-\left(\dot{P}^{g}\right)^{T} A^{g} \dot{P}^{g} \\
& -\left(\dot{P}^{l}\right)^{T} A^{l} \dot{P}^{l}-\left(C B \dot{\tilde{\phi}}-\dot{P}^{g}+\dot{P}^{l}\right)^{T}\left(C B \dot{\tilde{\phi}}-\dot{P}^{g}+\dot{P}^{l}\right) \\
& \leq 0
\end{aligned}
$$

Since $A^{g}, A^{l}$ and $D$ are positively definite diagonal matrices, $\dot{V}_{2}(w)=0$ holds only when $\dot{P}^{g}=\dot{P}^{l}=\dot{\omega}=0$. Therefore, for any $w(0) \in W_{2}^{*}$, the trajectory $w(t)$ satisfies

$$
\dot{P}^{g}(t)=\dot{P}^{l}(t)=\dot{\omega}(t)=0, \quad t \geq 0
$$

Hence $P^{g}(t), P^{l}(t)$ and $\omega(t)$ are all constants due to the boundedness property guaranteed by Lemma B.1.

On the other hand, for $\dot{V}_{2}(w)=0$, both terms in (B.3) have to be zero, implying that

$$
\hat{L}_{2}\left(w_{1}(t), w_{2}^{*}\right)=\hat{L}_{2}\left(w_{1}^{*}, w_{2}^{*}\right)
$$

must hold in $W_{2}$. Differentiating with respect to $t$ gives

$$
\left(\frac{\partial}{\partial w_{1}} \hat{L}_{2}\left(w_{1}(t), w_{2}^{*}\right)\right)^{T} \cdot \dot{w}_{1}(t)=0=-\dot{\tilde{\phi}}^{T}\left(\Gamma^{\tilde{\phi}}\right)^{-1} \dot{\tilde{\phi}}
$$

The second equality holds due to Eq. (B.5) and (13h). Then we can conclude $\dot{\tilde{\phi}}=0$ immediately, implying $\tilde{\phi}$ is also constant in $W^{*}$ due to its boundedness.

Invoking the close-loop dynamics (13), $\dot{\tilde{\theta}}(t), \dot{\eta}^{+}(t), \dot{\eta}^{-}(t)$ and $\dot{\lambda}(t)$ must be constants in $W_{2}^{*}$ as $P^{g}(t), P^{l}(t), \omega(t)$ and $\tilde{\phi}(t)$ are all constants. Then we conclude that $\dot{\tilde{\theta}}(t)=\dot{\eta}^{+}(t)=$ $\dot{\eta}^{-}(t)=\dot{\lambda}(t)=0$ holds for all $t \geq 0$ due to the boundedness property of $w(t)$ (Lemma B.1). This implies that any $w(0) \in$ $W_{2}^{*}$ must be an equilibrium point, completing the proof.

Proof of Theorem 4. Fix any initial state $w(0)$ and consider the trajectory $(w(t), t \geq 0)$ of the closed-loop system closeloop dynamics (13). As mentioned in the proof of Lemma B.2, $w(t)$ stays entirely in a compact set $\Omega_{0}$. Hence there exists an infinite sequence of time instants $t_{k}$ such that $w\left(t_{k}\right) \rightarrow \hat{w}^{*}$ as $t_{k} \rightarrow \infty$, for some $\hat{w}^{*} \in W_{2}^{*}$. Lemma B.2 guarantees that $\hat{w}^{*}$ is an equilibrium point of the closed-loop system (13), and hence $\hat{w}^{*}=H\left(\hat{w}^{*}\right)$. Thus, using this specific equlibrium point $\hat{w}^{*}$ in the definition of $V_{2}$, we have

$$
\begin{aligned}
V_{2}^{*}=\lim _{t \rightarrow \infty} V_{2}(w(t))=\lim _{t_{k} \rightarrow \infty} V_{2}\left(w\left(t_{k}\right)\right) \\
\quad=\lim _{w\left(t_{k}\right) \rightarrow \hat{w}^{*}} V_{2}\left(w\left(t_{k}\right)\right)=V_{2}\left(\hat{w}^{*}\right)=0
\end{aligned}
$$

Here, the first equality uses the fact that $V_{2}(t)$ is nonincreasing in $t$; the second equality uses the fact that $t_{k}$ is the infinite sequence of $t$; the third equality uses the fact that $w(t)$ is absolutely continuous in $t$; the fourth equality is due to the upper semi-continuity of $V_{2}(w)$, and the last equality holds as $\hat{w}^{*}$ is an equilibrium point of $V_{2}$.
The quadratic term $\left(w-\hat{w}^{*}\right)^{T} \Gamma_{2}^{-2}\left(w-\hat{w}^{*}\right)$ in $V_{2}$ then implies that $w(t) \rightarrow \hat{w}^{*}$ as $t \rightarrow \infty$, which completes the proof. 